\title{
TITLE:
}

\section{Quasinormal modes of D3-brane black holes}

AUTHOR(S):

Kurita, Y; Sakagami, M

\section{CITATION:}

Kurita, Y ...[et al]. Quasinormal modes of D3-brane black holes.

PHYSICAL REVIEW D 2003, 67(2): 024003.

ISSUE DATE:

2003-01-15

URL:

http://hdl.handle.net/2433/50234

RIGHT:

Copyright 2003 American Physical Society 
PHYSICAL REVIEW D 67, 024003 (2003)

\title{
Quasinormal modes of D3-brane black holes
}

\author{
Yasunari Kurita* \\ Graduate School of Human and Environmental Studies, Kyoto University, Kyoto 606-8501, Japan \\ Masa-aki Sakagami ${ }^{\dagger}$ \\ Department of Fundamental Sciences, FIHS, Kyoto University, Kyoto, 606-8501, Japan
}

(Received 9 August 2002; published 8 January 2003)

\begin{abstract}
We investigate a method to evaluate quasinormal modes of D3-brane black holes by wave interpretation of fields on a D3-brane based on Feynman's spacetime approach. We perturbatively solve the wave equation which describes propagation of a dilaton wave in a bulk space and its interaction with the D3-brane. The condition obtained for the quasinormal modes is qualitatively equivalent to that evaluated in the usual scattering of the dilaton in the black three-brane spacetime in the corresponding supergravity description.
\end{abstract}

DOI: 10.1103/PhysRevD.67.024003

PACS number(s): 04.70.-s, 04.50.+h, 11.25.Hf

\section{INTRODUCTION}

It is well known that black holes have thermodynamical properties, i.e., entropy [1,2] and temperature [3], which can be understood in the framework of general relativity [4] and the quantum theory of matter in curved spacetime [5]. In these formalisms, the entropy for a black hole is given by a quarter of its horizon area and Hawking radiation can be explained as particle creation caused by the existence of the horizon. Some years ago, in string theory, which seems to be the most promising candidate for a quantum theory of gravity, it was found that the D-brane can also describe black hole spacetime and its thermodynamical properties [6]. In this prescription, the entropy for a black hole is derived by counting the number of microscopic states on the D-brane [7], and Hawking radiation can be recognized as the emission process of closed strings from the D-brane $[8,9]$.

As for the scattering processes of a particle or a wave in black hole spacetime, we can observe good agreement between the D-brane picture and an analysis based on the corresponding supergravity description. In particular, the absorption cross section for a dilaton by the D3-brane in the low-energy region, which is evaluated by means of the world volume approach, has been shown to coincide with the result obtained by solving the wave equation for the dilaton field propagating in the three-brane background [10-12]. Taking account of the above results in the scattering processes, it seems to be quite natural to pose the question of whether the agreement holds or not even for quasinormal modes (QNMs) of a black hole.

QNMs characterize the emission of a gravitational wave which represents a response to a perturbation affecting a black hole spacetime [13-15]. As briefly reviewed in Appen$\operatorname{dix} \mathrm{A}$, it is obtained by solving the wave equation on a background with suitable boundary conditions that the flux at the horizon is ingoing and outgoing at spatial infinity. A QNM is characterized by a complex frequency whose imaginary part represents the time scale on which the perturbation to the

\footnotetext{
*Electronic address: kurita@phys.h.kyoto-u.ac.jp

${ }^{\dagger}$ Electronic address: sakagami@phys.h.kyoto-u.ac.jp
}

black hole spacetime decays. Since black holes are interpreted as thermal objects that are characterized by thermal quantities such as the temperature and entropy, the imaginary part of the QNM frequency can be recognized as the relaxation time within which black holes approach thermal equilibrium.

As is well known, the relationship between D-branes and black holes in string theory is an important precursor to the AdS conformal field theory (CFT) correspondence; and the connection between QNMs and the decay of perturbations in the dual CFT was first suggested in the work of [16] based on the numerical computation of QNMs for AdSSchwarzschild black holes in several dimensions. QNMs of AdS black holes and Bañados-Teitelboim-Zanelli (BTZ) black holes are investigated in [17-27] and those of near extremal black branes are found in [28]. Furthermore, in [29], it was shown that the frequencies of QNMs for BTZ black holes are in exact agreement with the location of the poles of the retarded correlation function describing the linear response on the CFT side.

In the present paper, we consider the D3-brane as another example of the correspondence in QNMs. Our analyses for D3-branes will be performed in two different parameter regions in type IIB string theory; one is a large number of the D3-branes and the other is only one D3-brane. In the first region, classical supergravity is effective so that gravity is described by the curvature of spacetime. In the latter, the perturbative field theory on the D3-brane which is embedded in a flat spacetime is effective. In such a flat spacetime, how can QNMs be calculated? This is our main interest and motivation in this paper. We will apply Feynman's spacetime approach to this problem, which gives alternative intuitive methods to quantum field theories [30-33]. We perturbatively solve the equation derived from the low-energy effective action for the D3-brane, i.e., the Dirac-Born-Infeld action, which describes propagation of a dilaton wave in the bulk flat spacetime and its interaction with D-branes. We apply a general condition for QNMs which is obtained in Appendix A to the scattering problem of this flat D3-brane case. It is shown that the condition obtained for QNMs is qualitatively equivalent to that evaluated in the framework of 
the usual scattering of the dilaton wave based on the supergravity description.

A brief outline of this paper follows. In the next section, we obtain the QNMs for the three-brane by solving the wave equation propagating on its background in classical supergravity analysis. In Sec. III, only one D3-brane is considered and the conditions for the QNMs are obtained by Feynman's spacetime approach. These two results are compared and discussed in Sec. IV. In Appendix A, we give a brief review of QNMs. Absorption of a scalar for the D3-brane is also investigated by the spacetime approach and the result is compared with the result of [11] in Appendix C.

\section{QUASINORMAL MODES IN SUPERGRAVITY}

In this section, we evaluate the QNM of the three-brane solution of type IIB supergravity. The main analysis of this section is based on the results obtained in [12], which studies the absorption probability of a dilaton by the D3-brane. We consider the dilaton as a minimally coupled scalar, which obeys the wave equation

$$
\frac{1}{\sqrt{-g}} \partial_{A} \sqrt{-g} g^{A B} \partial_{B} \phi=0 \quad(A, B=0,1, \ldots, 9)
$$

in the spacetime whose metric is given by

$$
\begin{aligned}
d s^{2}= & \left(1+\frac{R^{4}}{r^{4}}\right)^{-1 / 2}\left(-d t^{2}+d x_{i} d x^{i}\right)+\left(1+\frac{R^{4}}{r^{4}}\right)^{1 / 2} \\
& \times\left(d r^{2}+r^{2} d \Omega_{5}^{2}\right)
\end{aligned}
$$

The characteristic length of the three-brane $R$ is related to the ten-dimensional gravitational coupling constant $\kappa_{10}$ as follows:

$$
R^{4}=4 \pi g N \alpha^{\prime 2}=\frac{N \kappa_{10}}{2 \pi^{5 / 2}}
$$

In the case of the scattering in the low-energy region $\omega R \ll 1$, the dominant contribution to the cross section comes from the spherical symmetric process so that we concentrate on the radial equation for an $s$ wave of energy $\omega$ derived from Eq. (1):

$$
\left[\frac{\partial^{2}}{\partial r^{2}}+\frac{5}{r} \frac{\partial}{\partial r}+\omega^{2}\left(1+\frac{R^{4}}{r^{4}}\right)\right] \phi(r)=0 .
$$

If one performs the change of variables $r=R e^{-z}, \phi(r)$ $=e^{2 z} \psi(z)$, then Eq. (4) becomes the Mathieu equation:

$$
\left[\frac{\partial^{2}}{\partial z^{2}}+2(\omega R)^{2} \cosh 2 z-4\right] \psi(z)=0
$$

As was shown in [12], the exact solution which is ingoing at the horizon $(r \rightarrow 0)$ can be expressed as expansions in terms of Bessel and Hänkel functions as follows:

$$
\psi(z)=\sum_{n=-\infty}^{\infty} \frac{c(n+\mu)}{c(\mu)} J_{n}\left(\omega R e^{-z}\right) H_{n+2 \mu}^{(1)}\left(\omega R e^{z}\right),
$$

where the coefficients are given by

$$
\begin{aligned}
c(\mu) & =\frac{(\omega R / 2)^{2 \mu}}{\Gamma(\mu+2) \Gamma(\mu)} v(\mu), \\
v(\mu) & =\sum_{n=0}^{\infty}(-1)^{n}\left(\frac{\omega R}{2}\right)^{4 n} A_{\mu}^{(n)}, \\
A_{\mu}^{(0)} & =1, \\
A_{\mu}^{(q)} & =\sum_{p_{1}=0}^{\infty} \sum_{p_{2}=2}^{\infty} \ldots \sum_{p_{q}=2}^{\infty} a_{\mu+p_{1}} a_{\mu+p_{1}+p_{2}} \cdots \\
& \times a_{\mu+p_{1}+\cdots+p_{q},} \\
a_{\mu} & =\frac{1}{\mu(\mu+1)(\mu+2)(\mu+3)} .
\end{aligned}
$$

The value of $\mu$ is determined in terms of a prescription in the standard Floquet analysis which implies

$$
\frac{c(\mu)}{c(\mu-1)} \frac{c(-\mu+1)}{c(-\mu)}=1 .
$$

The explicit expression for the first few terms of $\mu$ and $A_{\mu}^{(q)}$ are given in Appendix B. Let me introduce new variables for convenience,

$$
\eta=e^{2 i \pi \mu}, \quad \chi=\frac{c(-\mu)}{c(\mu)}
$$

With these variables, the asymptotic form of the solution (6) near the horizon $(\operatorname{Re} z \rightarrow \infty)$ is given by

$$
\sqrt{\eta}\left(\eta-\frac{1}{\eta}\right) \psi(z) \rightarrow\left(\eta-\frac{1}{\eta}\right) \sqrt{\frac{2}{\pi \omega R e^{z}}} e^{i\left(\omega R e^{z}-\pi / 4\right)} .
$$

Similarly, we can obtain the asymptotic form for spatial infinity $(\operatorname{Re} z \rightarrow-\infty)$

$$
\begin{aligned}
\sqrt{\eta}\left(\eta-\frac{1}{\eta}\right) \psi(z) \rightarrow & \left(\chi-\frac{1}{\chi}\right) \sqrt{\frac{2}{\pi \omega R e^{-z}} e^{i\left(\omega R e^{-z}-\pi / 4\right)}} \\
& +\left(\eta \chi-\frac{1}{\eta \chi}\right) \sqrt{\frac{2}{\pi \omega R e^{-z}}} \\
& \times e^{-i\left(\omega R e^{-z}-\pi / 4\right)}
\end{aligned}
$$

From this asymptotic behavior given above, we can read off the amplitudes

$$
\mathcal{A}=\left(\eta-\frac{1}{\eta}\right), \quad \mathcal{I}=\left(\eta \chi-\frac{1}{\eta \chi}\right), \quad \mathcal{R}=\left(\chi-\frac{1}{\chi}\right),
$$


for the transmitted, incident, and reflected waves, respectively. As we describe in Appendix A, QNMs are given by the condition

$$
\mathcal{I} / \mathcal{R}=0
$$

In order to compare the results obtained (12) with the evaluation of QNMs in the next section based on the DiracBorn-Infeld action, which is the low-energy effective theory of the D3-branes, we consider the QNM condition (12) in the low-energy region, i.e., $\omega R \ll 1$, which can be expanded with respect to $\omega R$ as

$$
\begin{aligned}
\frac{\mathcal{I}}{\mathcal{R}} e^{i \beta}= & 1+\frac{\pi^{2}}{512}(\omega R)^{8}\left(1+\frac{4 i}{\pi} \log \frac{\omega \bar{R}}{2}\right)+\frac{\pi^{2}}{512 \cdot 12}(\omega R)^{12} \\
& \times\left[-2 \log \frac{\omega \bar{R}}{2}+\frac{4 i}{\pi}\left(\log \frac{\omega \bar{R}}{2}\right)^{2}\right]+\frac{\pi^{2}}{512} \frac{7}{72}(\omega R)^{12} \\
& \times\left(1+\frac{4 i}{\pi} \log \frac{\omega \bar{R}}{2}\right)+\mathcal{O}\left[(\omega R)^{16}(\log \omega R)^{3}\right]=0,
\end{aligned}
$$

where $\bar{R}$ is written as the characteristic length of the threebrane $R$ and the Euler constant $\gamma$ as $\bar{R}=e^{\gamma} R$, and $\beta$ is the phase factor

$$
\beta=\frac{2}{3} \pi\left(\frac{\omega R}{2}\right)^{4}+\frac{259}{216} \pi\left(\frac{\omega R}{2}\right)^{8}-\frac{22}{81} \pi^{3}\left(\frac{\omega R}{2}\right)^{12}+\cdots
$$

Let us solve the above QNM condition approximately up to the lowest order $(\omega R)^{8}$,

$$
1+\frac{\pi^{2}}{512}(\omega R)^{8}\left(1+\frac{4 i}{\pi} \log \frac{\omega \bar{R}}{2}\right)=0 .
$$

In the usual evaluation of QNMs [19-25,27], their frequencies are characterized by the curvature scale of the black hole spacetime, i.e., $|\omega R| \sim 1$. In contrasting, our calculation, which is valid in the range $|\omega R| \ll 1$, gives QNMs in the low-energy region. From the observational point of view, the frequencies for QNMs obtained in this paper might not be important. However, recall that our main interest is to confirm the equivalence between two different pictures, i.e., the D3-branes and black three-branes, in the case of QNMs.

From the condition (15) in the region $|\omega R| \ll 1$, we note that its solutions lie on the $n$th Riemanian sheets with $n \gg 1$ in the complex $\omega R$ plane. Inserting a polar coordinate representation for $(\omega R)^{8}$ into Eq. (15),

$$
(\omega R)^{8}=r e^{i \theta},
$$

we obtain two real equations,

$$
0=1+\frac{\pi^{2}}{2^{9}} r \cos \theta-\frac{\pi r}{2^{10}}\left[\left(\log r+8 \log \frac{e^{\gamma}}{2}\right) \sin \theta+\theta \cos \theta\right]
$$

$$
0=2 \pi \sin \theta+\left[\left(\log r+8 \log \frac{e^{\gamma}}{2}\right) \cos \theta-\theta \sin \theta\right] .
$$

From Eq. (18), $r$ is expressed in terms of $\theta$ as

$$
r=2^{8} e^{-8 \gamma} \exp [(\theta-2 \pi) \tan \theta] .
$$

So, we get the equation for $\theta$ from Eq. (17) and Eq. (19),

$$
1-\frac{\pi}{4 e^{8 \gamma}} e^{(\theta-2 \pi) \tan \theta} \frac{\theta-2 \pi}{\cos (\theta-2 \pi)}=0 .
$$

Since $r$ must satisfy $r \ll 1$, we can see from Eq. (17) that $\theta$ must satisfy $|\theta| \gg 1$. There are two cases for the solution of Eq. (19) and Eq. (20) as follows:

$$
\begin{aligned}
& \text { (i) } \theta>2 \pi, \quad \tan \theta<0, \quad \cos (\theta-2 \pi)>0 \\
& \Leftrightarrow \quad-\frac{\pi}{2}+2 n \pi<\theta<2 n \pi, \quad n \geqslant 2, \\
& \text { (ii) } \theta<2 \pi, \quad \tan \theta>0, \quad \cos (\theta-2 \pi)<0 \\
& \Leftrightarrow \quad-(2 n-1) \pi<\theta<-(2 n-1) \pi+\frac{\pi}{2}, \quad n \geqslant 0,
\end{aligned}
$$

where $n$ is an integer. The condition $|\theta| \gg 1$ is satisfied when $n \gg 1$. Let us consider the case (i). We put $\theta=2 n \pi-\Delta_{n}$ and $0<\Delta_{n}<\pi / 2$. Then Eq. (20) can be written as follows:

$$
1-\frac{\pi}{4 e^{8 \gamma}} e^{-2 n \pi \tan \Delta_{n}} \frac{2 n \pi}{\cos \Delta_{n}} \simeq 0
$$

$\Delta_{n}$ must be much less than unity in order that the second term of the above equation cancels the first term. It follows that

$$
\begin{gathered}
1-\frac{\pi}{4 e^{8 \gamma}} e^{-2 n \pi \Delta_{n} 2 n \pi \simeq 0} \\
\Rightarrow \Delta_{n} \simeq \frac{1}{2 n \pi}\left(\log n+\log \frac{\pi^{2}}{2 e^{8 \gamma}}\right) .
\end{gathered}
$$

In case (ii), we put $\theta=-(2 n+1) \pi+\Delta_{n}$. After a similar analysis, we have the same result Eq. (25) for $\Delta_{n}$. These $\Delta_{n}$ are very small number as long as $n \gg 1$, so that the solution of Eq. (15) is just below the real axis of the complex $(\omega R)^{8}$ plane. The radial parameter $r$ can be written as

$$
r=\frac{2^{9}}{\pi^{2} n}+\mathcal{O}\left(\frac{\log n}{n^{2}}\right)
$$

The QNM frequencies are $R^{-1}$ times the eighth roots of $r e^{i \theta}$. 


\section{THE WAVE SCATTERING OF THE D3-BRANE IN THE SPACETIME APPROACH}

In this section, we consider the case of a small number of D3-branes in string theory, so that the description of the system based on the field theory with Dirac-Born-Infeld (DBI) action is the effective one rather than that in terms of supergravity which was used in the previous section. For simplicity, we treat one D3-brane in flat spacetime and field theory with the DBI action on it. In the case of dilaton absorption by the D3-brane [10-12], the evaluation of the cross section is based on the world-volume interpretation, in which the bulk dilaton field is recognized as a source of the world-volume fluctuation. In other words, it is assumed that quantum fluctuation of the bulk fields decouples and the dynamics is strictly on the brane.

As explained in Appendix A, QNMs are determined by the condition (A10) which states that there is no incident wave in the scattering of the dilaton field in the black hole spacetime, and the reflective amplitude is needed to evaluate the QNM frequency. The world-volume approach [10-12] does not give the reflective amplitude which is concerned with bulk propagation. Thus the world-volume approach [10-12] is not suitable for investigation of QNMs. Since the above definition of QNMs (A10) is based on the scattering processes of the dilaton, we have to take account of its bulk propagation at least. In order to keep close similarity with the evaluation of QNMs in the previous section based on classical supergravity, we throw a dilaton into a D3-brane from spatial infinity, which is treated as a wave in field theory such as Feynman's spacetime approach [30-33]. The incident dilaton propagates in the bulk space and eventually interacts with the D3-brane. By perturbatively solving the wave equation for the dilaton, we can obtain its reflective amplitude. Another reason for using this spacetime approach is that we bear its waveform in mind, which is one of the other important aspects of QNMs. The calculation based on CFT [29] might be enough to evaluate the frequencies of QNMs which are given by the positions of the poles of the retarded CFT correlator. However, our approach can tell us the actual waveforms observed at infinity. Thus it is possible to discuss the relation between two pictures of black hole spacetime, i.e., the supergravity and the D-brane description, from the viewpoint not only of the frequencies of the QNMs but also of their waveforms. In Appendix $\mathrm{C}$, for a proof of the validity of our calculation based on the Feynman spacetime approach, the dilaton absorption cross section is evaluated by the formalism which is developed in the present paper. We show that our result agrees in the lowest order with that obtained by the world-volume approach [10-12].

For a low-energy dilaton, the DBI action can be expanded in the Einstein frame as follows [11]:

$$
\begin{aligned}
S_{\mathrm{DBI}}= & -T_{3} \int d^{4} x \sqrt{-\operatorname{det}\left(G_{\mu \nu}^{E}+\frac{e^{-\phi / 2}}{\sqrt{T_{3}}} F_{\mu \nu}\right)} \\
= & \int d^{4} x\left(\frac{1}{4} F^{2}-\frac{\sqrt{2}}{4} \kappa_{10} \phi F^{2}+\frac{1}{8 T_{3}}\left(F^{4}-\frac{1}{2}\left(F^{2}\right)^{2}\right)\right) \\
& +\cdots,
\end{aligned}
$$

where $F^{n}=F_{\mu 2}^{\mu 1} \cdots F_{\mu 1}^{\mu n}$ and $T_{3}$ is the tension of the D3brane. The term $F_{\mu \nu}$ is the field strength of the gauge field on the three-brane describing its longitudinal dynamics, while its transverse oscillations do not couple with the dilaton. For the $N=1$ case, the gauge fields on the D3-brane are photons, and we take the Coulomb gauge for them on the D3-brane. Then the action for the dilaton in the bulk and the gauge fields on the brane is given by

$$
\begin{aligned}
S_{\mathrm{eff}}= & -\frac{1}{2} \int d^{10} x \partial_{A} \phi \partial^{A} \phi-\frac{1}{2} \int d^{10} x \delta^{(6)}(x) \\
& \times\left[\left(1-\sqrt{2} \kappa_{10} \phi\right) \partial_{\mu} A_{i} \partial^{\mu} A^{i}+\frac{1}{2 T_{3}}\left(\epsilon^{i j k} \dot{A}_{i} \partial_{j} A_{k}\right)\right. \\
& \left.\times\left(\epsilon^{l m n} \dot{A}_{l} \partial_{m} A_{n}\right)\right],
\end{aligned}
$$

where $i=1,2,3$ and $\dot{A}=\partial_{0} A$. For a low energy dilaton, the scattering process is dominated by the $s$ wave so that we can put its form as $\phi=\phi(t, r)$ where $r^{2}=x_{4}^{2}+\cdots+x_{9}^{2}$. The evolution of the dilaton in the bulk and the gauge field on the brane are expressed by the wave equations

$$
\begin{gathered}
\left(-\partial_{t}^{2}+\partial_{r}^{2}-\frac{15}{4 r^{2}}\right) \varphi=-\delta(r) \frac{\kappa_{10}}{\sqrt{2} \pi^{3} r^{5 / 2}} \partial_{\mu} A_{i} \partial^{\mu} A^{i}, \\
\partial_{\mu} \partial^{\mu} A^{i}=\sqrt{2} \kappa_{10} \partial_{\mu}\left(\phi \partial^{\mu} A^{i}\right)+\frac{1}{T_{3}} \partial_{0}\left[\left(\epsilon^{l m n} \dot{A}_{l} \partial_{m} A_{n}\right) \epsilon^{i j k} \partial_{j} A_{k}\right] \\
+\frac{1}{T_{3}} \partial_{a}\left[\left(\epsilon^{l m n} \dot{A}_{l} \partial_{m} A_{n}\right) \epsilon^{b a i} \dot{A}_{b}\right],
\end{gathered}
$$

where we change the variable as $\varphi=r^{5 / 2} \phi$. We can solve these wave equations by means of perturbation with respect to interaction with the gauge fields, i.e., the coupling constant $\kappa_{10}$, and four-point interaction of the gauge fields, whose coupling constant is $1 / T_{3}$. First, these waves are formally expanded in terms of the two coupling constants,

$$
\begin{aligned}
\varphi= & \varphi_{(0,0)}+\kappa_{10} \varphi_{(1,0)}+\frac{\kappa_{10}}{T_{3}} \varphi_{(1,1)}+\kappa_{10}^{2} \varphi_{(2,0)} \\
& +\frac{\kappa_{10}^{2}}{T_{3}} \varphi_{(2,1)}+\cdots, \\
A_{i}= & A_{(0,0)}^{i}+\kappa_{10} A_{(1,0)}^{i}+\frac{1}{T_{3}} A_{(0,1)}+\frac{\kappa_{10}}{T_{3}} A_{(1,1)}^{i}+\cdots
\end{aligned}
$$

We are interested only in the response of the D3-brane to the incident dilaton wave. So we omit the terms $A_{(0,1)}$ and $\phi_{(1,0)}$ in the above expansions, since these terms represent the excitation which exists before the arrival of the injected dilaton at the three-brane.

At $(0,0)$ order, we obtain the wave equations 


$$
\begin{array}{r}
\left(-\partial_{t}^{2}+\partial_{r}^{2}-\frac{15}{4 r^{2}}\right) \varphi_{(0,0)}=0, \\
\partial_{\mu} \partial^{\mu} A_{(0,0)}^{i}=0,
\end{array}
$$

which express that the dilaton wave does not interact with the D3-brane at the origin $r=0$, and passes through it. So the regularity of the dilaton at the origin requires that the wave is reflected with the same amplitude as that of the incident wave. The solutions in the lowest order are given by

$$
\begin{aligned}
\varphi_{(0,0)}= & C e^{-i \omega t} \sqrt{r}\left[H_{2}^{(1)}(\omega r)+H_{2}^{(2)}(\omega r)\right] \\
A_{(0,0)}^{i}= & \int \frac{d^{3} k}{(2 \pi)^{3}} \sqrt{\frac{V_{3}}{2 k_{0}}}\left(\epsilon_{\mathbf{k}}^{\alpha}\right)^{i} e^{i \mathbf{k} \cdot \mathbf{x}}\left[e^{i k_{0} t} \sum_{\alpha=1}^{2} C_{\alpha}^{*}\right. \\
& \left.+e^{-i k_{0} t} \sum_{\alpha=1}^{2} C_{\alpha}\right]
\end{aligned}
$$

where $k_{0}=|\mathbf{k}|, \quad\left(\epsilon_{\mathbf{k}}^{\alpha}\right)^{i}$ is the polarization vector, and the complex number $C_{\alpha}$, which satisfies $\left|C_{\alpha}\right|^{2}=1$, represents a degree of freedom for the phase of the initial configuration of the gauge field. We prepare the wave function in the lowest order $A_{(0,0)}^{i}$ to express the vacuum fluctuation of the photon so that its normalization is taken to be the same as that in the corresponding quantum field theory.

At $(1,0)$ order, the wave equation for the gauge field is

$$
\partial_{\mu} \partial^{\mu} A_{(1,0)}^{i}=-\sqrt{2} \dot{\varphi}_{(0,0)} \dot{A}_{(0,0)}^{i}
$$

Here we choose a negative energy photon $A_{(0,0)}$ in Eq. (37). Thus this equation shows that the positive energy photon $A_{(1,0)}^{i}$ is created from the positive energy dilaton $\varphi_{(0,0)}$ and the negative energy photon $A_{(0,0)}^{i}$. Because, in the standard prescription of quantum field theories, a negative energy wave function is interpreted as an antiparticle which moves in the opposite direction, Eq. (37) describes a dilaton annihilation on the D3-brane and pair creation of photons like the calculation based on CFT in [10]. In order to construct the solution of Eq. (37), we use the retarded Green's function for the four-dimensional d'Alembertian,

$$
G_{A}\left(t, \mathbf{x} ; t^{\prime}, \mathbf{x}^{\prime}\right)=-\frac{\delta\left(t-t^{\prime}-\left|\mathbf{x}-\mathbf{x}^{\prime}\right|\right)}{4 \pi\left|\mathbf{x}-\mathbf{x}^{\prime}\right|},
$$

satisfying

$$
\partial_{\mu} \partial^{\mu} G_{A}\left(x ; x^{\prime}\right)=\delta^{(4)}\left(x-x^{\prime}\right)
$$

which leads to the photon created on the D3-brane,

$$
\begin{aligned}
A_{(1,0)}^{i}= & \frac{\sqrt{2} C \omega^{3}}{8} \int \frac{d^{3} k}{(2 \pi)^{3}} \sqrt{\frac{V_{3}}{2 k_{0}}} e^{-i\left(\omega-k_{0}\right) t} e^{i k_{i} x^{i}} \\
& \times\left(\frac{1}{\omega}+\frac{1}{2 k_{0}-\omega-i \epsilon}\right) \sum_{\alpha=1}^{2} C_{\alpha}^{*}\left(\epsilon_{k}^{\alpha}\right)^{i} .
\end{aligned}
$$

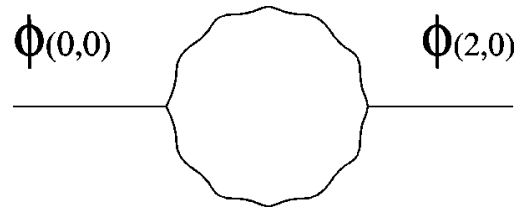

FIG. 1. The one-loop self-energy for the dilaton. Straight and wavy lines represent the propagating dilaton and photon, respectively. by

The wave equation for the dilaton at $(2,0)$ order is given

$$
\left(-\partial_{t}^{2}+\partial_{r}^{2}-\frac{15}{4 r^{2}}\right) \varphi_{(2,0)}=-\frac{2 \delta(r)}{\sqrt{2} \pi^{3} r^{5 / 2}} \partial_{\mu} A_{(0,0)}^{i} \partial^{\mu} A_{(1,0)}^{i} .
$$

The homogeneous part of the solution for the above equation should be zero, since there is no incident dilaton at $(2,0)$ order. Equation (41) represents the process that the excited gauge field $A_{(1,0)}^{i}$ and positive energy photon $A_{(0,0)}^{i}$ annihilate to emit a dilaton wave away from the D3-brane. We can construct the retarded Green's function for the dilaton,

$$
\begin{aligned}
G_{R}\left(t, r ; t^{\prime}, r^{\prime}\right)= & \frac{\pi}{2 i} \theta\left(t-t^{\prime}\right) \int \frac{d \omega}{2 \pi} e^{-i \omega\left(t-t^{\prime}\right)}\left[\theta\left(r-r^{\prime}\right)\right. \\
& \left.\times H_{2}^{(1)}(\omega r) J_{2}\left(\omega r^{\prime}\right) \sqrt{r r^{\prime}}+\left(r \leftrightarrow r^{\prime}\right)\right]
\end{aligned}
$$

which satisfies the equation

$$
\left(-\partial_{t}^{2}+\partial_{r}^{2}-\frac{15}{4 r^{2}}\right) G_{R}\left(t, r ; t^{\prime}, r^{\prime}\right)=\delta\left(t-t^{\prime}\right) \delta\left(r-r^{\prime}\right) .
$$

The explicit expression (43) shows that this Green's function is regular at the origin $r=0$ and obeys the outgoing condition at the spatial infinity $r \rightarrow \infty$. Then the $(2,0)$ dilaton which is integrated over the D3-brane is given by

$$
\begin{aligned}
\bar{\varphi}_{(2,0)} & =\frac{1}{V_{3}} \int_{V_{3}} d^{3} x \varphi_{(2,0)} \\
& =-\frac{C \omega^{8}}{32 \cdot 64 \pi^{3}} e^{-i \omega t} H_{2}^{(1)}(\omega r) \sqrt{r}\left(1+\frac{i}{\pi} \log \frac{\omega}{\Lambda}\right),
\end{aligned}
$$

where $\Lambda$ is a cutoff factor. Equation (41) tells us that $\bar{\varphi}_{(2,0)}$ is created through annihilation of two photons, $A_{(0,0)}^{i}$ and $A_{(1,0)}^{i}$, which are described by Eq. (37). As discussed before, it can be interpreted that two photons emerge via the pair creation process. Thus, in the framework of quantum field theories, the external line corrected by the one-loop selfenergy Feynman diagram depicted in Fig. 1 would correspond to the dilaton wave function $\bar{\varphi}_{(2,0)}$. We note that the field $A_{(0,0)}$ appearing in Eq. (37) and that in Eq. (41) are identical to those shown in Fig. 1, although we interpret 
these two photons as a particle-antiparticle pair. Thus, in the evaluation of Eq. (44), momentum integrations are doubly counted so that we have introduced a symmetric factor $1 / 2$. The divergent contribution to the $(2,0)$-order dilaton should be removed by the renormalization procedure in which the cutoff $\Lambda$ is replaced by a scale for renormalization $R$, i.e., the characteristic length of the black hole.

At $(1,1)$ order, the equation for $\varphi_{(1,1)}$ has no source terms and is not created. The equation for $A_{(1,1)}^{i}$ is given by

$$
\begin{aligned}
\partial_{\mu} \partial^{\mu} A_{(1,1)}^{i}= & \partial_{0}\left[\dot{\mathbf{A}}_{(1,0)} \cdot\left(\nabla \times \mathbf{A}_{(0,0)}\right) \epsilon_{k}^{i j} \partial_{j} A_{(0,0)}^{k}\right] \\
& +\partial_{a}\left[\dot{\mathbf{A}}_{(1,0)} \cdot\left(\nabla \times \mathbf{A}_{(0,0)}\right) \epsilon^{b a i} \dot{A}_{(0,0) b}\right] \\
& +\partial_{0}\left[\dot{\mathbf{A}}_{(0,0)} \cdot\left(\nabla \times \mathbf{A}_{(1,0)}\right) \epsilon_{k}^{i j} \partial_{j} A_{(0,0)}^{k}\right] \\
& +\partial_{a}\left[\dot{\mathbf{A}}_{(0,0)} \cdot\left(\nabla \times \mathbf{A}_{(1,0)}\right) \epsilon^{b a i} \dot{A}_{(0,0) b}\right] \\
& +\partial_{0}\left[\dot{\mathbf{A}}_{(0,0)} \cdot\left(\nabla \times \mathbf{A}_{(0,0)}\right) \epsilon_{k}^{i j} \partial_{j} A_{(1,0)}^{k}\right] \\
& +\partial_{a}\left[\dot{\mathbf{A}}_{(0,0)} \cdot\left(\nabla \times \mathbf{A}_{(0,0)}\right) \epsilon^{b a i} \dot{A}_{(1,0) b}\right] .
\end{aligned}
$$

This equation describes a four-point interaction of photons. As in the case of the $(1,0)$-order photon $(37)$, one of the $(0,0)$ photons $A_{(0,0)}^{i}$ is chosen as the negative energy photon in the right-hand side so that Eq. (45) can be interpreted as two-photon scattering. The scattered $(1,1)$-order photon $A_{(1,1)}$ and $(0,0)$-order photon $A_{(0,0)}^{i}$ are annihilated to produce a $(2,1)$-order dilaton as follows:

$$
\left(-\partial_{t}^{2}+\partial_{r}^{2}-\frac{15}{4 r^{2}}\right) \varphi_{(2,1)}=-\delta(r) \frac{\sqrt{2}}{\pi^{3} r^{5 / 2}} \partial_{\mu} A_{(0,0) i} \partial^{\mu} A_{(1,1)}^{i} .
$$

The source term of this equation is integrated over the D3brane to give

$$
\begin{aligned}
\frac{1}{V_{3}} \int d^{3} x \partial_{\mu} A_{(0,0) i} \partial^{\mu} A_{(1,1)}^{i} & \\
= & \frac{C \omega^{10}}{3 \cdot 2^{11} \sqrt{2} \pi^{4}} e^{-i \omega t}\left[\pi^{2}+2 \pi i \log \frac{\omega}{\Lambda}-\left(\log \frac{\omega}{\Lambda}\right)^{2}\right] .
\end{aligned}
$$

Using the Green's function for a dilaton Eq. (43), we obtain the solution of Eq. (46) integrated over the D3-brane,

$$
\begin{aligned}
\bar{\varphi}_{(2,1)} & =\frac{1}{V_{3}} \int_{V_{3}} d^{3} x \varphi_{(2,1)} \\
& =-\int d t^{\prime} d r^{\prime} G_{R} \frac{\sqrt{2} \delta\left(r^{\prime}\right)}{\pi^{3} r^{\prime 5 / 2}} \frac{1}{V_{3}} \int d^{3} x \partial_{\mu} A_{(0,0) i} \partial^{\mu} A_{(1,1)}^{i} \\
& =C \frac{\omega^{12} e^{-i \omega t}}{3 \cdot 2^{14} \pi^{4}} H_{2}^{(1)}(\omega r) \sqrt{r}\left[i-\frac{2}{\pi} \log \frac{\omega}{\Lambda}-\frac{i}{\pi^{2}}\left(\log \frac{\omega}{\Lambda}\right)^{2}\right],
\end{aligned}
$$

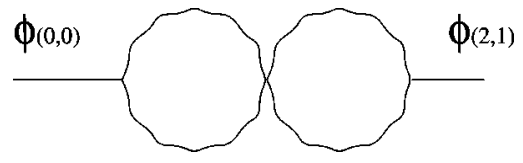

FIG. 2. The two-loop self-energy for the dilaton. Straight and wavy lines represent the propagating dilaton and photon, respectively.

where we again introduce a symmetric factor $1 / 4$, since the double counting in the momentum integration, which emerged in the evaluation of Eq. (44), occurs twofold in this case. The cutoff $\Lambda$ will be replaced by the inverse of the characteristic length of the black hole $R^{-1}$ by the procedure of renormalization. The wave function $\bar{\varphi}_{(2,1)}$ obtained corresponds to the external line corrected by the two-loop Feynman diagram depicted in Fig. 2.

Assembly of the calculated dilaton waves at several orders leads to

$$
\begin{aligned}
\bar{\varphi} & =\bar{\varphi}_{(0,0)}+\kappa_{10}^{2} \bar{\varphi}_{(2,0)}+\frac{\kappa_{10}^{2}}{T_{3}} \bar{\varphi}_{(2,1)}+\cdots \\
& =C e^{-i \omega t} \sqrt{r}\left[\mathcal{R} H_{2}^{(1)}(\omega r)+H_{2}^{(2)}(\omega r)\right] \\
& \rightarrow C e^{-i \omega t} \sqrt{\frac{2}{\pi \omega}}\left[\mathcal{R} e^{i(\omega r-54 \pi / 4)}+e^{-i(\omega r-5 \pi / 4)}\right],
\end{aligned}
$$

where in the last line we take the $r \rightarrow \infty$ limit and $\mathcal{R}$ is the reflective amplitude,

$$
\begin{aligned}
\mathcal{R}= & 1-\frac{\kappa_{10}^{2} \omega^{8}}{2^{11} \pi^{3}}\left(1+\frac{i}{\pi} \log \omega R\right)+\frac{\kappa_{10}^{2}}{T_{3}} \frac{\omega^{12}}{3 \cdot 2^{18} \pi^{4}} \\
& \times\left(i-\frac{2}{\pi} \log \omega R-\frac{i}{\pi^{2}}(\log \omega R)^{2}\right)+\cdots
\end{aligned}
$$

Here, we recall the relation between $\kappa_{10}, T_{3}$, and $R$ :

$$
\kappa_{10}=\frac{\sqrt{\pi}}{T_{3}}, \quad R^{4}=\frac{\kappa_{10}}{2 \pi^{5 / 2}}
$$

where we set $N=1$. Thus the condition of the QNM discussed in Appendix A is given by

$$
\begin{aligned}
0= & \frac{\mathcal{I}}{\mathcal{R}} e^{i \beta^{\prime}} \\
= & 1+\frac{\pi^{2}}{512}(\omega R)^{8}\left(1+\frac{i}{\pi} \log \omega R\right)+\frac{\pi^{2}}{512 \cdot 12}(\omega R)^{12} \\
& \times\left(\log \omega R+\frac{i}{2 \pi}(\log \omega R)^{2}\right)+\mathcal{O}\left[(\omega R)^{16}\right],
\end{aligned}
$$

where $\beta^{\prime}$ is a phase factor, 


$$
\beta^{\prime}=\frac{\pi^{3}}{512 \cdot 24}(\omega R)^{12}
$$

Equation (54) up to $(\omega R)^{8}$ is very similar to Eq. (15), and the solution has qualitatively equivalent behavior.

\section{DISCUSSION}

We have evaluated quasinormal modes of the D3-brane black hole by two different approaches. One is based on the black hole picture in terms of the black three-brane solution of the low-energy supergravity action and the other is the D3-brane described by the field theory with the Dirac-BornInfeld action. We have shown that the two different methods derive qualitatively the same conditions for the QNMs for the low-energy region. As described in Appendix C, the evaluations of the absorption cross sections show similar agreement. In both cases, the difference, which appears in the logarithmic terms, seems to imply that we must take proper account of the non-Abelian nature of the theory $[11,12,34]$.

The condition for QNMs (A10) has been obtained as Eq. (13) and Eq. (54) in terms of an expansion with respect to $\omega R$. Up to the order $(\omega R)^{8}$, we can express the condition for the QNM as follows:

$$
\frac{\mathcal{I}}{\mathcal{R}} \sim 1+i \Sigma=0
$$

where $\sim$ means equality up to phase factors and

$$
\Sigma=\frac{\pi^{2}}{512}(\omega R)^{8}\left(\frac{\alpha}{\pi} \log \frac{\omega \bar{R}}{2}-i\right) .
$$

In the field theoretical approach as in Sec. III, $\Sigma$, which is just the coefficient of $\phi_{(2,0)}$, corresponds to the one-loop self-energy of the dilaton. The procedure of the spacetime approach gives the dilaton wave function as Eq. (51), and we can read off the reflective amplitude (52) which is expressed as

$$
\frac{\mathcal{R}}{\mathcal{I}} \sim 1-i \Sigma
$$

up to the order $(\omega R)^{8}$. In our calculation this reflective amplitude is perturbatively inverted to give Eq. (56). However, from the viewpoint of an evaluation based on field theories, the above inversion can be interpreted as the summation of the self-energy diagrams

$$
\frac{\mathcal{R}}{\mathcal{I}} \sim 1+(-i \Sigma)+(-i \Sigma)^{2}+\cdots \sim \frac{1}{1+i \Sigma} .
$$

Thus QNMs can be understood as poles of the scattered propagator in the field theory on the D3-brane. This is realized in the calculation of the AdS/CFT correspondence in the BTZ black hole case in [29].

\section{ACKNOWLEDGMENT}

We would like to thank T. Uematsu and S. Yamaguchi for useful discussions.

\section{APPENDIX A: A BRIEF REVIEW OF QNMS}

In this appendix, we give a brief review of quasinormal modes which represent the relaxation process in a black hole spacetime. If one perturbs the black hole spacetime, then a gravitational wave is emitted to both directions. One part of the wave propagates to spatial infinity and the other falls into the black hole horizon. So it is natural to specify the boundary condition for such waves as follows: ingoing at the horizon and outgoing at spatial infinity.

Let us consider a minimal scalar on the black hole background. It satisfies a wave equation of the following form after some change of variables [15]:

$$
\left[-\partial_{t}^{2}+\partial_{r_{*}}^{2}-V\left(r_{*}\right)\right] \phi=0
$$

where $r_{*}$ is the "tortoise" radial coordinate. In the case of the Schwarzschild black hole with the horizon radius $r$ $=r_{h}$, the coordinate $r_{*}$ is related to the usual radial coordinate $r$ as

$$
r_{*}=r+r_{h} \ln \left(\frac{r-r_{h}}{r_{h}}\right),
$$

which spans the region outside the horizon of the black hole. It follows that $r_{*} \rightarrow-\infty$ represents approaching the horizon $\left(r=r_{h}\right)$ and $r_{*} \rightarrow \infty$ the spatial infinity $(r \rightarrow \infty)$, respectively. The potential $V\left(r_{*}\right)$ which has the information of the curvature of the spacetime, is assumed to have the asymptotic behavior, $V\left(r_{*}\right) \rightarrow 0$ as $r_{*} \rightarrow-\infty$ (to the horizon) and $r_{*}$ $\rightarrow \infty$ (to spatial infinity). Under this assumption, the solution of wave equation (A1) with frequency $\omega$ can be expressed as

$$
\phi\left(t, r_{*}\right)=A e^{-i \omega\left(t-r_{*}\right)}+B e^{-i \omega\left(t+r_{*}\right)}
$$

in the asymptotic region. Here let me remind you the boundary conditions, i.e., QNMs are ingoing at the horizon and outgoing at the spatial infinity, which are specified by

$$
\phi \propto \begin{cases}e^{-i \omega\left(t-r_{*}\right)} & \left(r_{*} \rightarrow \infty\right), \\ e^{-i \omega\left(t+r_{*}\right)} & \left(r_{*} \rightarrow-\infty\right) .\end{cases}
$$

In order to obtain a solution which satisfies the boundary condition described above, we consider the retarded Green's function constructed by the following prescription. We prepare two solutions $\phi_{1}\left(t, r_{*}\right)$ and $\phi_{2}\left(t, r_{*}\right)$, which have the asymptotic forms

$$
\begin{gathered}
\phi_{1} \sim \begin{cases}\mathcal{I} e^{-i \omega\left(t+r_{*}\right)}+\mathcal{R} e^{-i \omega\left(t-r_{*}\right)} & \left(r_{*} \rightarrow \infty\right), \\
\mathcal{A} e^{-i \omega\left(t+r_{*}\right)} & \left(r_{*} \rightarrow-\infty\right),\end{cases} \\
\phi_{2} \sim \begin{cases}\mathcal{Q} e^{-i \omega\left(t-r_{*}\right)} & \left(r_{*} \rightarrow \infty\right), \\
\mathcal{P} e^{-i \omega\left(t-r_{*}\right)}+\mathcal{G} e^{-i \omega\left(t+r_{*}\right)} & \left(r_{*} \rightarrow-\infty\right),\end{cases}
\end{gathered}
$$


to construct the retarded Green's function from them,

$$
\begin{aligned}
G_{R}\left(t, r_{*} ; t^{\prime}, r_{*}^{\prime}\right)= & \theta\left(t-t^{\prime}\right) \int \frac{d \omega}{2 \pi} e^{-i \omega\left(t-t^{\prime}\right)} \\
& \times \frac{1}{\Delta}\left[\theta\left(r_{*}-r_{*}^{\prime}\right) \phi_{1}\left(r_{*}^{\prime}\right) \phi_{2}\left(r_{*}\right)\right. \\
& \left.+\left(r_{*} \leftrightarrow r_{*}^{\prime}\right)\right],
\end{aligned}
$$

where the Wronskian $\Delta$ can be evaluated as $\Delta=2 i \omega \mathcal{I} \mathcal{Q}$ from the asymptotic forms at $r_{*} \rightarrow \infty$. Using this retarded Green's function, we can obtain the wave $\phi$ generated by a source term $S\left(t, r_{*}\right)$ :

$$
\phi\left(t, r_{*}\right)=\int d t^{\prime} d r_{*}^{\prime} G_{R}\left(t, r_{*} ; t^{\prime}, r_{*}^{\prime}\right) S\left(t^{\prime}, r_{*}^{\prime}\right)
$$

which satisfies the inhomogeneous equation

$$
\left[-\partial_{t}^{2}+\partial_{r_{*}}^{2}-V\left(r_{*}\right)\right] \phi=S\left(t, r_{*}\right) .
$$

It is obvious that the solution (A7) satisfies the QNM boundary condition (A3). Taking the limit $r_{*} \rightarrow \infty$ of Eqs. (A7) and (A6), we obtain the asymptotic form of the generated wave as

$$
\begin{aligned}
\phi\left(t, r_{*}\right) \rightarrow & \int d t^{\prime} d r_{*}^{\prime} \theta\left(t-t^{\prime}\right) \\
& \times \int_{\mathrm{C}} \frac{d \omega}{2 \pi} \frac{e^{-i \omega\left(t-t^{\prime}\right)} e^{i \omega r_{*}}}{2 i \omega \mathcal{I}} \phi_{1}\left(r_{*}^{\prime}\right) S\left(t^{\prime}, r_{*}^{\prime}\right),
\end{aligned}
$$

where the contour $\mathrm{C}$ must be taken as it encloses the lower half of the complex $\omega$ plane for the retarded boundary condition. So only the poles of the lower half plane contribute to the $\omega$ integral in the asymptotic form Eq. (A9) and that is the quasinormal modes which are, of course, complex and decaying modes. In other words, QNMs are the poles of the retarded Green's function on the lower half complex $\omega$ plane. The condition for the QNM is $\mathcal{I}=0$, but only the ratio of amplitudes is meaningful in the asymptotic form Eq. (A4), so this is equivalent to $\mathcal{R} \rightarrow \infty$. Then the more general condition for the QNM is

$$
\mathcal{I} / \mathcal{R}=0
$$

The only thing we have to do is to solve this equation and choose modes arising in the lower half of the complex plane.

\section{APPENDIX B: EXPLICIT EXPRESSIONS}

In this appendix, we summarize the explicit forms for some results which were considered too lengthy to write out in the main text.

The Floquet exponent $\mu$ which satisfies Eq. (8) can be obtained in the expansion with respect to a power series in $\omega R$,

$$
\begin{aligned}
\mu= & 1-\frac{i \sqrt{5}}{6}\left(\frac{\omega R}{2}\right)^{4}+\frac{7 i}{216 \sqrt{5}}\left(\frac{\omega R}{2}\right)^{8}+\frac{11851 i}{62208 \sqrt{5}}\left(\frac{\omega R}{2}\right)^{12} \\
& +\cdots
\end{aligned}
$$

The coefficients $A_{\mu}^{(q)}$ in Eq. (7) up to $(\omega R)^{16}$ are given by

$$
\begin{aligned}
& A_{\mu}^{(1)}=\frac{1}{3 \mu(\mu+1)(\mu+2)}, \\
& A_{\mu}^{(2)}=\frac{1}{432}\left(\frac{1}{\mu}-\frac{11}{\mu+1}-\frac{47}{\mu+2}\right) \\
& -\frac{1}{144} \frac{1}{\mu+3}-\frac{1}{18} \frac{1}{(\mu+2)^{2}} \\
& +\frac{5}{36} \psi^{(1)}(\mu+2), \\
& A_{\mu}^{(3)}=-\frac{2321}{155520 \mu}+\frac{739}{9720(\mu+1)}-\frac{5}{216(\mu+2)^{3}} \\
& -\frac{115}{2592(\mu+2)^{2}}-\frac{791}{9720(\mu+2)}+\frac{1}{432(\mu+3)^{2}} \\
& +\frac{61}{6480(\mu+3)}+\frac{1}{17280(\mu+4)}+\left(\frac{7}{648}+\frac{5}{216 \mu}\right. \\
& \left.-\frac{5}{108(\mu+1)}+\frac{5}{216(\mu+2)}\right) \psi^{(1)}(\mu+2), \\
& A_{\mu}^{(4)}=\left(\frac{164327}{133996800}-\frac{37 \pi^{2}}{31104}\right) \frac{1}{\mu+1}+\cdots,
\end{aligned}
$$

where $\psi^{(1)}$ is the polyGamma function.

\section{APPENDIX C: ABSORPTION CROSS SECTION FOR D3-BRANE}

In this appendix, we compare the absorption cross section $\sigma$ for the D3-brane in various methods of evaluation. As shown in [11], there is a small difference in $\sigma$ for different methods at higher order.

In supergravity, the absorption cross section $\sigma$ for the three-brane is given by

$$
\begin{aligned}
\sigma_{\text {SUGRA }} & =\frac{32 \pi^{2}}{\omega^{5}} \frac{|\mathcal{A}|^{2}}{|\mathcal{I}|^{2}} \\
& =\frac{\pi^{4}}{8} \omega^{3} R^{8}\left(1-\frac{1}{6}(\omega R)^{4} \log \omega \bar{R}+\mathcal{O}\left[(\omega R)^{4}\right]\right)
\end{aligned}
$$

from Eq. (11) as in [12]. This evaluation is reliable in the $N \gg 1$ region where the supergravity description of the threebrane is valid. 
We can also calculate the absorption cross section $\sigma$ for the D3-brane by means of the space-time approach from Eq. (51):

$$
\begin{aligned}
\sigma_{\text {wave }} & =\frac{32 \pi^{2}}{\omega^{5}}\left(1-\left|\frac{\mathcal{R}}{\mathcal{I}}\right|^{2}\right) \\
& =\frac{\pi^{4}}{8} \omega^{3} R^{8}\left(1+\frac{1}{12}(\omega R)^{4} \log \frac{\omega}{\Lambda}+\mathcal{O}\left[(\omega R)^{4}\right]\right),
\end{aligned}
$$

where we set $N=1$.

As in [11], CFT on the D3-brane can also be used to derive this quantity, and the result is

$$
\sigma_{\mathrm{CFT}}=\frac{\pi^{4}}{8} \omega^{3} R^{8}\left(1-\frac{1}{24}(\omega R)^{4} \log \frac{\omega}{\Lambda}+\mathcal{O}\left[(\omega R)^{4}\right]\right) .
$$

These three evaluations of $\sigma$ are agree at the lowest order.
[1] J. D. Bekenstein, Phys. Rev. D 5, 1239 (1972).

[2] S. W. Hawking, Phys. Rev. Lett. 26, 1344 (1971).

[3] S. W. Hawking, Commun. Math. Phys. 43, 199 (1975).

[4] For example, R. M. Wald, General Relativity (University of Chicago Press, Chicago, 1984).

[5] For example, N. D. Birrell and P. C. W. Davies, Quantum Fields in Curved Space (Cambridge University Press, Cambridge, England, 1982).

[6] See, e.g, G. Horowitz, gr-qc/9604051.

[7] A. Strominger and C. Vafa, Phys. Lett. B 379, 99 (1996).

[8] C. G. Callan and J. M. Maldacena, Nucl. Phys. B472, 591 (1996).

[9] S. R. Das and S. D. Mathur, Nucl. Phys. B478, 561 (1996).

[10] I. R. Klebanov, Nucl. Phys. B496, 231 (1997).

[11] S. S. Gubser, A. Hashimoto, I. R. Klebanov, and M. Krasnitz, Nucl. Phys. B526, 393 (1998).

[12] S. S. Gubser and A. Hashimoto, Commun. Math. Phys. 203, 325 (1999).

[13] S. Chandrasekhar and S. Detweiler, Proc. R. Soc. London A344, 441 (1975).

[14] See, e.g., S. Chandrasekhar, The Mathematical Theory of Black Holes (Clarendon, Oxford, 1983).

[15] See e.g., K. D. Kokkotas and B. G. Schmidt, Living Rev. Relativ. 2, 2 (1999).

[16] G. T. Horowitz and V. E. Hubeny, Phys. Rev. D 62, 024027 (2000).

[17] J. S. F. Chan and R. B. Mann, Phys. Rev. D 55, 7546 (1997).
[18] J. S. F. Chan and R. B. Mann, Phys. Rev. D 59, 064025 (1999).

[19] B. Wang, C. Lin, and E. Abdalla, Phys. Lett. B 481, 79 (2000).

[20] T. R. Govindarajan and V. Suneeta, Class. Quantum Grav. 18, 265 (2001).

[21] Jiong-Ming Zhu, Bin Wang, and Elcio Abdalla, Phys. Rev. D 63, 124004 (2001).

[22] Danny Birmingham, Phys. Rev. D 64, 064024 (2001).

[23] Vitor Cardoso and José P. S. Lemos, Phys. Rev. D 64, 084017 (2001).

[24] Vitor Cardoso and José P. S. Lemos, Class. Quantum Grav. 18, 5257 (2001).

[25] Ian G. Moss and James P. Norman, Class. Quantum Grav. 19, 2323 (2002).

[26] B. Wang, E. Abdalla, and R. B. Mann, Phys. Rev. D 65, 084006 (2002).

[27] R. A. Konoplya, Phys. Rev. D 66, 044009 (2002); 66, 084007 (2002).

[28] Andrei O. Starinets, Phys. Rev. D 66, 124013 (2002).

[29] D. Birmingham, I. Sachs, and S. N. Solodukhin, Phys. Rev. Lett. 88, 151301 (2002).

[30] R. P. Feynman, Rev. Mod. Phys. 21, 425 (1949).

[31] R. P. Feynman, Rev. Mod. Phys. 20, 367 (1948).

[32] R. P. Feynman, Phys. Rev. 76, 749 (1949).

[33] J. J. Sakurai, Advanced Quantum Mechanics (Addison-Wesley, Reading, MA, 1967), Chaps. 4 and 5.

[34] C. G. Callan, S. S. Gubser, I. R. Klebanov, and A. A. Tseytlin, Nucl. Phys. B489, 65 (1997). 